\title{
NUMERICAL APPROACH FOR THE FREQUENCY-WEIGHTED HANKEL-NORM APPROXIMATION
}

\author{
Andras Varga \\ German Aerospace Center, DLR - Oberpfaffenhofen \\ Institute of Robotics and Mechatronics \\ D-82234 Wessling, Germany. \\ fax: $\quad+49-8153-28-1441$ \\ e-mail: Andras.Varga@dlr.de
}

Keywords: model reduction, frequency-weighting, Hankelnorm approximation, numerical methods.

\begin{abstract}
We derive new projection formulas for the model reduction method based on the frequency-weighted Hankel norm approximation (FWHNA). These formulas extend the applicability of the FWHNA method to frequency weights expressed as antistable right/left invertible rational matrices. By computing the projections via the solution of appropriate generalized Sylvester equations, an inversion-free solution of the FWHNA problem is possible. The new projection formulas allows to implement efficiently the FWHNA method as robust numerical software. We also discuss the solution of the frequencyweighted $L_{\infty}$-norm model reduction problem and indicate how to solve it in the most general setting.
\end{abstract}

\section{Introduction}

Consider the $n$-th order original state-space model $\mathbf{G}:=$ $(A, B, C, D)$ with the transfer-function matrix (TFM)

$$
G(\lambda)=C(\lambda I-A)^{-1} B+D,
$$

and let $\mathbf{G}_{r}:=\left(A_{r}, B_{r}, C_{r}, D_{r}\right)$ be an $r$-th order approximation of the original model $(r<n)$, with the TFM

$$
G_{r}(\lambda)=C_{r}\left(\lambda I-A_{r}\right)^{-1} B_{r}+D_{r} .
$$

According to the system type, $\lambda$ is either the complex variable $s$ appearing in the Laplace transform in the case of a continuoustime system or the variable $z$ appearing in the $Z$-transform in the case of a discrete-time system.

Notation. Throughout the paper we use the bold-notation $\mathbf{G}$ to denote a state-space system having the TFM $G(\lambda)$ or $G$. This notation is used consistently to denote systems corresponding to particular TFMs: GK denotes the series coupling of two systems having the TFM $G(\lambda) K(\lambda), \mathbf{G}^{-1}$ denotes the inverse system corresponding to the inverse TFM $G^{-1}(\lambda)$, and $\mathbf{G}^{\sim}$ denotes the conjugate system corresponding to the conjugate TFM $G^{\sim}(\lambda)$, where $G^{\sim}(s)=G^{T}(-s)$ for a continuous-time system and $G^{\sim}(z)=G^{T}(1 / z)$ for a discrete-time system.

The Hankel-norm approximation (HNA) method [4] belongs to the class of absolute (or additive) error model reduction methods and relies on a guaranteed error bound. Glover [4] has shown that for a stable $\mathbf{G}$, there exists an $r$-th order stable approximation $\mathbf{G}_{r}$ such that

$$
\left\|G-G_{r}\right\|_{H}=\sigma_{r+1}(\mathbf{G})
$$

where $\sigma_{r+1}(\mathbf{G})$ is the $(r+1)$-th largest Hankel singular value of $\mathbf{G}$. Note that, because the Hankel-norm is only a seminorm, the choice of $D_{r}$ in (1) plays no role on the achieved optimal Hankel-norm of the approximation error.

The frequency-weighted HNA (FWHNA) problem has been originally formulated in [7] to minimize the weighted-error

$$
\left\|W_{o}^{\sim}\left(G-G_{r}\right) W_{i}^{\sim}\right\|_{H}
$$

where $\mathbf{W}_{o}$ and $\mathbf{W}_{i}$ are systems whose TFMs $W_{o}$ and $W_{i}$ represent suitable output and input weighting, respectively. The presence of weights usually reflects the desire that the approximation be more accurate at those frequencies where $W_{o}$ and $W_{i}$ have larger singular values. The standard assumptions in [7] are: $W_{o}$ and $W_{i}$ are biproper, stable and minimum-phase TFMs. A solution of the FWHNA problem for scalar systems has been proposed by Latham and Anderson in [7] and extended to the multivariable case by Hung and Glover in [5] .

In this paper we consider the alternative formulation of the FWHNA problem to minimize

$$
\left\|W_{o}\left(G-G_{r}\right) W_{i}\right\|_{H}
$$

for which the corresponding standard assumptions become: $W_{o}$ and $W_{i}$ are biproper TFMs, having only unstable poles and zeros. In this case, the optimal frequency-weighted approximation error satisfies [7, 5]

$$
\left\|W_{o}\left(G-G_{r}\right) W_{i}\right\|_{H}=\sigma_{r+1}\left(\mathbf{G}_{1}\right),
$$

where $\mathbf{G}_{1}:=\left[\mathbf{W}_{o} \mathbf{G} \mathbf{W}_{i}\right]_{+}$is the stable projection of the system $\mathbf{W}_{o} \mathbf{G} \mathbf{W}_{i}$.

An efficient algorithm to solve the FWHNA problem in either forms (2) or (3) has been derived by the author in [12]. This algorithm is based on explicit projection formulas and avoids the forming of state space realizations of the form $\mathbf{W}_{o}^{\sim} \mathbf{G W}_{i}^{\sim}$ or $\mathbf{W}_{o} \mathbf{G W}_{i}$. To compute the projections, appropriate continuous- or discrete-time Sylvester equations are solved. 
Several practical problems, as for example some controller reduction problems, do not fit into the standard formulations above. This is why, the assumptions on weights have been relaxed in several ways. In what follows, we only discuss the FWHNA formulation (3), but all considerations can be easily adapted to the formulation (2). The first extension, done in [5], allows $W_{o}$ and $W_{i}$ to have arbitrary zeros, which however must be different from the poles of $\mathbf{G}_{1 r}$, the HNA of $\mathbf{G}_{1}$ ( this is automatically satisfied when both $W_{o}$ and $W_{i}$ have only unstable zeros). The projection formulas derived in [12] can be employed also for this case.

The assumption for biproper weights has been relaxed to proper and right/left invertible weights by Zhou [17]. However, the suggested computational solution has only a theoretical value, because it involves the reduction of the state matrix of $\mathbf{G}_{1 r}$ to the Jordan canonical form. In general, this reduction relies on using possibly ill-conditioned non-orthogonal similarity transformations and thus, can not be performed in a numerically reliable way.

In this paper we develop a numerically reliable computational approach to solve the FWHNA problem with antistable weights $W_{o}$ and $W_{i}$, such that $W_{o}$ is full row rank and $W_{i}$ is full column rank. The procedure has the following main steps:

\section{FWHNA Procedure}

1. Compute the $n$-th order stable projection

$$
\mathbf{G}_{1}=\left[\mathbf{W}_{o} \mathbf{G} \mathbf{W}_{i}\right]_{+}
$$

2. Compute $\mathbf{G}_{1 r}$, the optimal $r$-th order HNA of $\mathbf{G}_{1}$.

3. Compute $\mathbf{G}_{r}$, the $r$-th order projection of $\mathbf{W}_{o}^{R} \mathbf{G}_{1 r} \mathbf{W}_{i}^{L}$ containing the poles of $G_{1 r}$, where $\mathbf{W}_{o}^{R}$ is a right-inverse of $\mathbf{W}_{o}$ and $\mathbf{W}_{i}^{L}$ is a left-inverse of $\mathbf{W}_{i}$.

This procedure is applicable for $W_{o}$ and $W_{i}$ having arbitrary zeros, provided the finite zeros of $W_{o}$ and $W_{i}$ are distinct from the poles of $G_{1 r}$, the optimal HNA computed at step 2 of the above procedure. With obvious replacements, the same procedure can be employed to solve the FWHNA problem (2).

For an efficient implementation of the FWHNA Procedure, we derive new projection formulas based on descriptor system descriptions. These formulas allow an inversion-free implementation of the FWHNA Procedure, for both formulations (2) and (3) of the FWHNA problem. The proposed computational solution of the FWHNA problem represents a general numerically reliable alternative to the procedure proposed in [17]. Interestingly, our approach can be employed even if the weights are improper, a possibility also mentioned in [17].

We also address the solution of the frequency-weighted $L_{\infty}$-norm model reduction problem by using the FWHNA method. Specifically, we consider the computation of an $L_{\infty^{-}}$ optimal feedthrough matrix $D_{r}$ and discuss methods to convert non-standard problems to the standard-form required by the FWHNA Procedure.

\section{Projection formulas for $\mathbf{W}_{o} \mathbf{G} \mathbf{W}_{i}$}

We assume that $W_{o}$ and $W_{i}$ are antistable TFMs with descriptor realizations of the form

$$
\begin{aligned}
& \mathbf{W}_{o}=\left(A_{o}-\lambda E_{o}, B_{o}, C_{o}, D_{o}\right) \\
& \mathbf{W}_{i}=\left(A_{i}-\lambda E_{i}, B_{i}, C_{i}, D_{i}\right)
\end{aligned}
$$

satisfying

$$
\begin{aligned}
& W_{o}(\lambda)=C_{o}\left(\lambda E_{o}-A_{o}\right)^{-1} B_{o}+D_{o} \\
& W_{i}(\lambda)=C_{i}\left(\lambda E_{i}-A_{i}\right)^{-1} B_{i}+D_{i}
\end{aligned}
$$

We also assume that $\mathbf{G}=(A, B, C, D)$ is stable and thus has all its poles distinct from those of $\mathbf{W}_{o}$ and $\mathbf{W}_{i}$. We can easily construct the system

$$
\mathbf{W}_{o} \mathbf{G W}_{i}:=\left(A_{w}-\lambda E_{w}, B_{w}, C_{w}, D_{w}\right)
$$

where

$$
\begin{aligned}
A_{w}-\lambda E_{w} & =\left[\begin{array}{ccc}
A_{o}-\lambda E_{o} & B_{o} C & B_{o} D C_{i} \\
0 & A-\lambda I & B C_{i} \\
0 & 0 & A_{i}-\lambda E_{i}
\end{array}\right], \\
B_{w} & =\left[\begin{array}{c}
B_{o} D D_{i} \\
B D_{i} \\
B_{i}
\end{array}\right] \\
C_{w} & =\left[\begin{array}{lll}
C_{o} & D_{o} C & D_{o} D C_{i}
\end{array}\right] \\
D_{w} & =D_{o} D D_{i}
\end{aligned}
$$

Let $U$ and $V$ be the transformation matrices defined by

$$
U=\left[\begin{array}{ccc}
I & -E_{o} X & 0 \\
0 & I & -Y \\
0 & 0 & I
\end{array}\right], \quad V=\left[\begin{array}{ccc}
I & X & 0 \\
0 & I & Y E_{i} \\
0 & 0 & I
\end{array}\right]
$$

where $X$ and $Y$ satisfy the generalized Sylvester equations

$$
\begin{array}{r}
A_{o} X-E_{o} X A+B_{o} C=0 \\
A Y E_{i}-Y A_{i}+B C_{i}=0
\end{array}
$$

Since the generalized eigenvalues of the pairs $\left(A_{o}, E_{o}\right)$ and $\left(A_{i}, E_{i}\right)$ are distinct from the eigenvalues of $A$, each of the equations (5) and (6) has always a unique solution. When $W_{o}$ and $W_{i}$ are proper rational matrices, and $E_{o}=I, E_{i}=I$, then the generalized Sylvester equations (5) and (6) become standard Sylvester equations.

It is straightforward to check that

$$
\begin{aligned}
U\left(A_{w}-\lambda E_{w}\right) V & =\left[\begin{array}{ccc}
A_{o}-\lambda E_{o} & 0 & * \\
0 & A-\lambda I & 0 \\
0 & 0 & A_{i}-\lambda E_{i}
\end{array}\right], \\
U B_{w} & =\left[\begin{array}{c}
* \\
B D_{i}-Y B_{i} \\
B_{i}
\end{array}\right] \\
C_{w} V & =\left[\begin{array}{ll}
C_{o} & C_{o} X+D_{o} C *
\end{array}\right]
\end{aligned}
$$


where $*$ denotes arbitrary matrices not important in the present context. Thus $\mathbf{W}_{o} \mathbf{G} \mathbf{W}_{i}$ can be additively decomposed uniquely as

$$
\mathbf{W}_{o} \mathbf{G} \mathbf{W}_{i}=\mathbf{G}_{1}+\mathbf{G}_{2}
$$

where $\mathbf{G}_{1}$ and $\mathbf{G}_{2}$ are given by

$$
\begin{aligned}
\mathbf{G}_{1} & =\left(A, B D_{i}-Y B_{i}, C_{o} X+D_{o} C, D_{o} D D_{i}\right) \\
\mathbf{G}_{2} & =\left(\left[\begin{array}{cc}
A_{o}-\lambda E_{o} & * \\
0 & A_{i}-\lambda E_{i}
\end{array}\right],\left[\begin{array}{c}
* \\
B_{i}
\end{array}\right],\left[C_{o} *\right], 0\right)
\end{aligned}
$$

In (7), $\mathbf{G}_{1}$ can be generally seen as the projection of $\mathbf{W}_{o} \mathbf{G} \mathbf{W}_{i}$ which contains the poles of $\mathbf{G}$.

\section{Projection formulas for $\mathbf{W}_{o}^{R} \mathbf{G} \mathbf{W}_{i}^{L}$}

We assume that $W_{o}$ is full row rank and $W_{i}$ is full column rank to ensure the existence of a right inverse $W_{o}^{R}$ such that $W_{o} W_{o}^{R}=I$ and of a left inverse $W_{i}^{L}$ such that $W_{i}^{L} W_{i}=I$. Having explicit realizations for $\mathbf{W}_{o}^{R}$ and $\mathbf{W}_{i}^{L}$ we can apply the projection formulas of previous section to compute the projection necessary at step 3 of the FWHNA Procedure. In what follows we discuss only the computation of a right inverse for $W_{o}$. However, the approach which we present is also applicable to compute a left inverse of $W_{i}$ by computing a right inverse for $W_{i}^{T}$ (the dual of $W_{i}$ ).

We consider first the case of a square invertible $W_{o}$. If $D_{o}$ is invertible, we can form the explicit inverse

$$
\mathbf{W}_{o}^{-1}=\left(A_{o}-B_{o} D_{o}^{-1} C_{o}-\lambda E_{o},-B_{o} D_{o}^{-1}, D_{o}^{-1} C_{o}, D_{o}^{-1}\right)
$$

The main advantage in the FWHNA Procedure of using the explicit inverses is that the resulting feedthrough matrix of $G_{1 r}$ at step 3 appears in the expression of $D_{r}$.

If $W_{o}$ is invertible as a rational matrix, but $D_{o}$ is ill-conditioned or singular, then we can employ the implicit form of the inverse

$$
\mathbf{W}_{o}^{-1}=\left(\left[\begin{array}{cc}
A_{o} & B_{o} \\
C_{o} & D_{o}
\end{array}\right]-\lambda\left[\begin{array}{cc}
E_{o} & 0 \\
0 & 0
\end{array}\right],\left[\begin{array}{l}
0 \\
I
\end{array}\right],\left[\begin{array}{ll}
0 & -I
\end{array}\right], 0\right)
$$

in conjunction with the developed projection formulas. The main advantage if this approach is the inversion-free formulation. A possible disadvantage of using implicit inverses in the FWHNA Procedure is that the resulting feedthrough matrix $D_{r}$ at step 3 is always zero. Since the Hankel-norm is only a seminorm, this has no consequence on the Hankel-norm approximation error. Still, if we use the FWHNA to obtain a good approximation by minimizing the $L_{\infty}$-norm of the weighted error, then $D_{r}$ must be determined separately by solving a convex optimization problem as suggested in [17].

If $\mathbf{W}_{o}$ is non-square, an appropriate right inverse $\mathbf{W}_{o}^{R}$ must be determined such that $\mathbf{W}_{o}^{R}$ and $\mathbf{G}_{1 r}$ have no common poles. In what follows we present a computational approach derived from the more general technique to determine weak generalized inverses proposed by the author in [15]. It is straightforward to show that for a $p \times m$ full row rank rational matrix $W_{o}$, a right inverse can be computed using the formula

$$
W_{o}^{R}(\lambda)=\left[\begin{array}{ll}
0 & I_{m}
\end{array}\right] S_{o}^{R}(\lambda)\left[\begin{array}{c}
0 \\
I_{p}
\end{array}\right]
$$

where $S_{o}(\lambda)$ is the full row rank system pencil

$$
S_{o}(\lambda)=\left[\begin{array}{cc}
A_{o}-\lambda E_{o} & B_{o} \\
C_{o} & D_{o}
\end{array}\right]
$$

By using the above formula, the computation of a right-inverse of $W_{o}$ can be accomplished by computing a right-inverse of the associated system pencil.

The computation of $S_{o}^{R}(\lambda)$ can be done by reducing $S_{o}(\lambda)$ to an appropriate Kronecker-like form from which a maximal rank regular sub-pencil can be easily separated. Let $Q$ and $Z$ be orthogonal matrices to reduce $S_{o}(\lambda)$ to the Kronecker-like form

$$
\bar{S}_{o}(\lambda):=Q S_{o}(\lambda) Z=\left[\begin{array}{c|cc}
B_{r} & A_{r}-\lambda E_{r} & A_{r, z}-\lambda E_{r, z} \\
0 & 0 & A_{z}-\lambda E_{z}
\end{array}\right]
$$

where the regular part $A_{z}-\lambda E_{z}$ contains the finite and infinite zeros of $W_{o}$, and the pair $\left(A_{r}-\lambda E_{r}, B_{r}\right)$ is controllable with $E_{r}$ nonsingular (see [13] for how to obtain such a Kroneckerlike form). The controllability of the pair $\left(A_{r}-\lambda E_{r}, B_{r}\right)$ is the consequence of the full row rank assumption on $W_{o}$.

It is easy to see that a right inverse can be defined as

$$
\mathbf{W}_{o}^{R}:=\left(\bar{A}_{12}-\lambda \bar{E}_{12}, \bar{B}_{1}, \bar{C}_{2}, 0\right),
$$

where

$$
\begin{gathered}
\bar{A}_{12}-\lambda \bar{E}_{12}:=\left[\begin{array}{cc}
A_{r}-\lambda E_{r} & A_{r, z}-\lambda E_{r, z} \\
0 & A_{z}-\lambda E_{z}
\end{array}\right], \\
{\left[\begin{array}{c}
\bar{B}_{1} \\
\bar{B}_{2}
\end{array}\right]:=Q\left[\begin{array}{c}
0 \\
I_{p}
\end{array}\right], \quad\left[\bar{C}_{1} \bar{C}_{2}\right]:=\left[0-I_{m}\right] Z}
\end{gathered}
$$

The eigenvalues of the right inverse are

$$
\Lambda\left(\bar{A}_{12}, \bar{E}_{12}\right)=\Lambda_{\text {fixed }} \cup \Lambda_{\text {spurious }},
$$

where $\Lambda_{\text {fixed }}=\Lambda\left(A_{z}, E_{z}\right)$ are the eigenvalues of the regular part and thus, contain the system zeros, and $\Lambda_{\text {spurious }}=$ $\Lambda\left(A_{r}, E_{r}\right)$ are the finite "spurious" zeros originating from the column singularity of $W_{o}(\lambda)$.

While the system zeros are always among the poles of the right inverse, the spurious poles can be arbitrarily chosen. To show this, consider a transformation matrix $V$ of the form

$$
V=\left[\begin{array}{c|cc}
I & F_{r} & 0 \\
\hline 0 & I & 0 \\
0 & 0 & I
\end{array}\right]
$$

Then, the transformed system pencil is given by

$$
\widehat{S}(\lambda):=\bar{S}_{o}(\lambda) V=\left[\begin{array}{c|cc}
B_{r} & A_{r}+B_{r} F_{r}-\lambda E_{r} & A_{r, z}-\lambda E_{r, z} \\
0 & 0 & A_{z}-\lambda E_{z}
\end{array}\right]
$$


A right inverse can be defined this time as

$$
\mathbf{W}_{o}^{R}:=\left(\widehat{A}_{12}-\lambda \widehat{E}_{12}, \widehat{B}_{1}, \widehat{C}_{2}, 0\right),
$$

where

$$
\begin{aligned}
& \widehat{A}_{12}-\lambda \widehat{E}_{12}:=\left[\begin{array}{cc}
A_{r}+B_{r} F_{r}-\lambda E_{r} & A_{r, z}-\lambda E_{r, z} \\
0 & A_{z}-\lambda E_{z}
\end{array}\right], \\
& {\left[\begin{array}{c}
\widehat{B}_{1} \\
\widehat{B}_{2}
\end{array}\right]:=Q\left[\begin{array}{c}
0 \\
I_{p}
\end{array}\right], \quad\left[\widehat{C}_{1} \widehat{C}_{2}\right]:=\left[0-I_{m}\right] Z V .}
\end{aligned}
$$

To obtain a right inverse with all spurious poles lying in the antistable domain of the complex plane $\mathbb{C}_{a}$, we choose $F_{r}$ such that $\Lambda\left(A_{r}+B_{r} F_{r}, E_{r}\right) \subset \mathbb{C}_{a}$ by solving a standard pole assignment problem (with $E_{r}$ nonsingular). This is always possible because the pair $\left(A_{r}-\lambda E_{r}, B_{r}\right)$ is controllable.

\section{Projection formulas for $\mathrm{W}_{o}^{\sim} \mathbf{G W}_{i}^{\sim}$}

The projection formulas are dependent on the type of the system. Therefore, we derive distinct formulas for continuoustime and discrete-time systems. In this section, we assume that $\mathbf{G}, \mathbf{W}_{o}$ and $\mathbf{W}_{i}$ are stable.

\subsection{Continuous-time system}

For a continuous-time system we have the following explicit formulas for $W_{o}^{\sim}$ and $W_{i}^{\sim}$

$$
\begin{aligned}
& \mathbf{W}_{o}^{\sim}=\left(-A_{o}^{T}-\lambda E_{o}^{T},-C_{o}^{T}, B_{o}^{T}, D_{o}^{T}\right) \\
& \mathbf{W}_{i}^{\sim}=\left(-A_{i}^{T}-\lambda E_{i}^{T},-C_{i}^{T}, B_{i}^{T}, D_{i}^{T}\right)
\end{aligned}
$$

Using the results of section 2 , we obtain the required projection of $\mathbf{W}_{o}^{\sim} \mathbf{G} \mathbf{W}_{i}^{\sim}$ as

$$
\mathbf{G}_{1}=\left(A, B D_{i}^{T}+Y C_{i}^{T}, B_{o}^{T} X+D_{o}^{T} C, D_{o}^{T} D D_{i}^{T}\right)
$$

where $X$ and $Y$ satisfy the generalized Sylvester equations

$$
\begin{aligned}
A_{o}^{T} X+E_{o}^{T} X A+C_{o}^{T} C & =0 \\
A Y E_{i}^{T}+Y A_{i}^{T}+B B_{i}^{T} & =0
\end{aligned}
$$

\subsection{Discrete-time system}

To derive the analogous discrete-time formulas, we can use explicit state space realizations for $\mathbf{W}_{o}^{\sim}$ and $\mathbf{W}_{i}^{\sim}$ only in the case when the corresponding state matrices $A_{o}$ and $A_{i}$ are invertible. Therefore, we derive the projection formulas avoiding the explicit inversion of these matrices.

To simplify the presentation, we derive the projection in two steps: first we determine the projection $\widetilde{\mathbf{G}}_{1}$ of $\mathbf{W}_{o}^{\sim} \mathbf{G}$ and then the projection $\mathbf{G}_{1}$ of $\widetilde{\mathbf{G}}_{1} \mathbf{W}_{i}^{\sim}$. Using the expression of $W_{o}^{\sim}(z)$

$$
W_{o}^{\sim}(z)=B_{o}^{T}\left(z^{-1} E_{o}^{T}-A_{o}^{T}\right)^{-1} C_{o}^{T}+D_{o}^{T}
$$

we can compute

$$
\begin{aligned}
W_{o}^{\sim}(z) G(z)= & B_{o}^{T}\left(z^{-1} E_{o}^{T}-A_{o}^{T}\right)^{-1} C_{o}^{T} C(z I-A)^{-1} B \\
& +B_{o}^{T}\left(z^{-1} E_{o}^{T}-A_{o}^{T}\right)^{-1} C_{o}^{T} D \\
& +D_{o}^{T} C(z I-A)^{-1} B+D_{o}^{T} D
\end{aligned}
$$

Let $X$ be the solution of the generalized Sylvester equation

$$
E_{o}^{T} X-A_{o}^{T} X A=C_{o}^{T} C
$$

and consider the straightforward identity

$$
\begin{array}{r}
\left(z^{-1} E_{o}^{T}-A_{o}^{T}\right) X(z I-A)+\left(z^{-1} E_{o}^{T}-A_{o}^{T}\right) X A \\
+A_{o}^{T} X(z I-A)=E_{o}^{T} X-A_{o}^{T} X A
\end{array}
$$

Combining (10) and (11), we can express the first term of $W_{o}^{\sim}(z) G(z)$ as

$$
\begin{array}{r}
B_{o}^{T}\left(z^{-1} E_{o}^{T}-A_{o}^{T}\right)^{-1} C_{o}^{T} C(z I-A)^{-1} B= \\
B_{o}^{T} X B+B_{o}^{T} X A(z I-A)^{-1} B+ \\
B_{o}^{T}\left(z^{-1} E_{o}^{T}-A_{o}^{T}\right)^{-1} A_{o}^{T} X B
\end{array}
$$

It follows that the projection $\widetilde{\mathbf{G}}_{1}$ containing the poles of $\mathbf{G}$ can be expressed as

$$
\widetilde{\mathbf{G}}_{1}=\left(A, B, D_{o}^{T} C+B_{o}^{T} X A, B_{o}^{T} X B+D_{o}^{T} D\right)
$$

Similarly, we can derive the expression of projection $\widehat{\mathbf{G}}_{1}$ of $\mathbf{G W}_{i}^{\sim}$

$$
\widehat{\mathbf{G}}_{1}=\left(A, B D_{i}^{T}+A Y C_{i}^{T}, C, D D_{i}^{T}+C Y C_{i}^{T}\right)
$$

where $Y$ satisfies the generalized Sylvester equation

$$
Y E_{i}^{T}-A Y A_{i}^{T}=B B_{i}^{T}
$$

Applying the above approach to compute the projection $\mathbf{G}_{1}$ of $\widetilde{\mathbf{G}}_{1} \mathbf{W}_{i}^{\sim}$, we get

$$
\mathbf{G}_{1}=\left(A, B D_{i}^{T}+A Y C_{i}^{T}, D_{o}^{T} C+B_{o}^{T} X A, D_{1}\right)
$$

where

$$
D_{1}=D_{o}^{T} D D_{i}^{T}+B_{o}^{T} X B D_{i}^{T}+D_{o}^{T} C Y C_{i}^{T}+B_{o}^{T} X A Y C_{i}^{T}
$$

Note that for proper $\mathbf{W}_{o}$ and $\mathbf{W}_{i}$ with $E_{o}=I, E_{i}=I$, the generalized Sylvester equations (10) and (12) become the discrete Sylvester equations derived in [12].

\section{Projection formulas for $\left(\mathbf{W}_{o}^{\sim}\right)^{R} \mathbf{G}\left(\mathbf{W}_{i}^{\sim}\right)^{L}$}

The projection of $\left(\mathbf{W}_{o}^{\sim}\right)^{R} \mathbf{G}\left(\mathbf{W}_{i}^{\sim}\right)^{L}$ containing the poles of $\mathbf{G}$ can be obtained by applying the appropriate approach in Section 4 to compute the projection of $\left(\mathbf{W}_{o}^{R}\right) \sim \mathbf{G}\left(\mathbf{W}_{i}^{L}\right) \sim$. Appropriate right and left inverses can be computed using the methods described in Section 3.

\section{Numerical aspects}

In this section we give an implementable, inversion-free version of the FWHNA Procedure based on the projection formulas derived in Section 2. We assume $\mathbf{G}=(A, B, C, D)$ stable, $\mathbf{W}_{o}=\left(A_{o}-\lambda E_{o}, B_{o}, C_{o}, D_{o}\right)$ antistable, with $W_{o}(\lambda)$ full row rank and $\mathbf{W}_{i}=\left(A_{i}-\lambda E_{i}, B_{i}, C_{i}, D_{i}\right)$ antistable with $W_{i}(\lambda)$ full column rank. Further, we tacitly assume that the HNA $\mathbf{G}_{1 r}$ computed at Step 2 of the FWHNA Procedure has no poles which are zeros of either $\mathbf{W}_{o}$ or $\mathbf{W}_{i}$. 


\section{FWHNA Algorithm}

1. Compute an orthogonal transformation matrix $Q$ to reduce $A$ to the real Schur form (RSF) and compute

$$
A \leftarrow Q^{T} A Q, \quad B \leftarrow Q^{T} B, \quad C \leftarrow C Q
$$

2. Compute orthogonal matrices $Q_{o}$ and $Z_{o}$ to reduce the pair $\left(A_{o}, E_{o}\right)$ to the generalized real Schur form (GRSF) and compute

$$
A_{o}-\lambda E_{o} \leftarrow Q_{o}^{T}\left(A_{o}-\lambda E_{o}\right) Z_{o}, B_{o} \leftarrow Q_{o}^{T} B_{o}, C_{o} \leftarrow C_{o} Z_{o}
$$

3. Solve for $X$ and $U$ the Sylvester system

$$
\begin{array}{lll}
A_{o} X-U A+B_{o} C & =0 \\
E_{o} X-U & = & 0
\end{array}
$$

4. Compute orthogonal matrices $Q_{i}$ and $Z_{i}$ to reduce the pair $\left(A_{i}, E_{i}\right)$ to the GRSF and compute

$$
A_{i}-\lambda E_{i} \leftarrow Q_{i}^{T}\left(A_{i}-\lambda E_{i}\right) Z_{i}, B_{i} \leftarrow Q_{i}^{T} B_{i}, C_{i} \leftarrow C_{i} Z_{i}
$$

5. Solve for $V$ and $Y$ the Sylvester system

$$
\begin{array}{ll}
A V-Y A_{i}+B C_{i} & =0 \\
V-Y E_{i} & =0
\end{array}
$$

6. Compute an $r$-th order approximation

$$
\mathbf{G}_{1 r}=\left(A_{r}, B_{1 r}, C_{1 r}, D_{1 r}\right)
$$

of the system

$$
\mathbf{G}_{1}=\left(A, B D_{i}-Y B_{i}, C_{o} X+D_{o} C, D_{o} D D_{i}\right)
$$

using the HNA method [4].

7. Form a right inverse system $\mathbf{W}_{o}^{R}=\left(\widetilde{A}_{o}-\lambda \widetilde{E}_{o}, \widetilde{B}_{o}, \widetilde{C}_{o}, \widetilde{D}_{o}\right)$.

8. Compute orthogonal matrices $\widetilde{Q}_{o}$ and $\widetilde{Z}_{o}$ to reduce the pair $\left(\widetilde{A}_{o}, \widetilde{E}_{o}\right)$ to the GRSF and compute

$$
\widetilde{A}_{o}-\lambda \widetilde{E}_{o} \leftarrow \widetilde{Q}_{o}^{T}\left(\widetilde{A}_{o}-\lambda \widetilde{E}_{o}\right) \widetilde{Z}_{o}, \widetilde{B}_{o} \leftarrow \widetilde{Q}_{o}^{T} \widetilde{B}_{o}, \widetilde{C}_{o} \leftarrow \widetilde{C}_{o} \widetilde{Z}_{o}
$$

9. Solve for $\widetilde{X}$ and $\widetilde{U}$ the Sylvester system

$$
\begin{array}{ll}
\widetilde{A}_{o} \widetilde{X}-\widetilde{U} A_{r}+\widetilde{B}_{o} C_{1 r} & =0 \\
\widetilde{E}_{o} \widetilde{X}-\widetilde{U} & =0
\end{array}
$$

10. Form a left inverse system $\mathbf{W}_{i}^{L}=\left(\widetilde{A}_{i}-\lambda \widetilde{E}_{i}, \widetilde{B}_{i}, \widetilde{C}_{i}, \widetilde{D}_{i}\right)$.

11. Compute orthogonal matrices $\widetilde{Q}_{i}$ and $\widetilde{Z}_{i}$ to reduce the pair $\left(\widetilde{A}_{i}, E_{i}\right)$ to the GRSF and compute

$$
\widetilde{A}_{i}-\lambda \widetilde{E}_{i} \leftarrow \widetilde{Q}_{i}^{T}\left(\widetilde{A}_{i}-\lambda \widetilde{E}_{i}\right) \widetilde{Z}_{i}, \widetilde{B}_{i} \leftarrow \widetilde{Q}_{i}^{T} \widetilde{B}_{i}, \widetilde{C}_{i} \leftarrow \widetilde{C}_{i} \widetilde{Z}_{i}
$$

12. Solve for $\widetilde{V}$ and $\widetilde{Y}$ the Sylvester system

$$
\begin{array}{ll}
A_{r} \widetilde{V}-\widetilde{Y} \widetilde{A}_{i}+B_{1 r} \widetilde{C}_{i} & =0 \\
\widetilde{V}-\widetilde{Y} \widetilde{E}_{i} & =0
\end{array}
$$

13. Compute the $r$-th order reduced model

$$
\mathbf{G}_{r}=\left(A_{r}, B_{1 r} \widetilde{D}_{i}-\widetilde{Y} \widetilde{B}_{i}, \widetilde{C}_{o} \widetilde{X}+\widetilde{D}_{o} C_{1 r}, \widetilde{D}_{o} D_{1 r} \widetilde{D}_{i}\right)
$$

At Steps 3, 5, 9 and 12, the generalized Schur method is used to solve the Sylvester systems [6]. These systems arise from an equivalent reformulation of the respective generalized Sylvester equations. When $W_{o}$ and $W_{i}$ are proper, then the Schur method [2] can be used to solve these equations as standard Sylvester equations. Note that in both cases the reduction of $A$ to the RSF is necessary, being also the first step when performing the HNA at Step 6. Because the last computation in the HNA algorithm of [4] is an additive stable-unstable spectral separation, the resulting $A_{r}$ can be assumed to be already in a RSF (used to reorder the eigenvalues), thus no further reduction of $A_{r}$ is necessary.

If the feedthrough matrices $D_{o}$ and $D_{i}$ are invertible and well conditioned, then the explicit inverses of $W_{o}$ and $W_{i}$ can be used, without any practically significant accuracy loss. The advantage of using explicit inverses is that the resulting $D_{r}$ is related to the HNA computed at Step 6. For an efficient implementation of the FWHNA method, it is possible to further refine the computation of projections, by exploiting all structural features of the problem. For example, if $D_{o}$ is square and well-conditioned, and $D_{i}$ is square but ill-conditioned or singular, it is possible to compute the projection of $\mathbf{W}_{o}^{-1} \mathbf{G}_{1 r} \mathbf{W}_{i}^{-1}$ by employing an explicit inverse for $\mathbf{W}_{o}^{-1}$ and the implicit inverse for $\mathbf{W}_{i}^{-1}$.

To solve (2), completely similar procedures for continuoustime and discrete-time systems can be used for the FWHNA method based on the projection formulas developed in Section 4 . The only difference appears in the discrete-time case for proper weights, where the projection formulas involves the solution of discrete Sylvester equations for which algorithms proposed in [11] can be used.

\section{Weighted $L_{\infty}$-norm model reduction}

One of the main usage of the FWHNA method is in solving the frequency-weighted $L_{\infty}$-approximation problem. The FWHNA can be used to produce good approximation errors in the $L_{\infty}$-norm, which satisfy

$$
\sigma_{r+1}\left(\mathbf{G}_{1}\right) \leq\left\|W_{o}\left(G-G_{r}\right) W_{i}\right\|_{\infty}
$$

where the best $D_{r}$ can be determined by a convex optimization [17]. For the computation of an optimal $D_{r}$ and possibly $C_{r}$ or $B_{r}$, recently developed fast algorithms can be employed [16].

For the $L_{\infty}$-norm approximation, we only need to assume that the weights have no poles on the imaginary axis in continuoustime or on the unit circle in discrete-time. In the case when $W_{o}$ and/or $W_{i}$ have stable poles, then left/right coprime factorizations with antistable all-pass denominators can be determined for $W_{o}$ and $W_{i}$ using the methods proposed in [14, 9]. If $W_{o}=M_{o}^{-1} N_{o}$ and $W_{i}=N_{i} M_{i}^{-1}$ are the respective factorizations with $N_{o}$ and $N_{i}$ antistable, then

$$
\left\|W_{o}\left(G-G_{r}\right) W_{i}\right\|_{\infty}=\left\|N_{o}\left(G-G_{r}\right) N_{i}\right\|_{\infty}
$$


It was empirically observed that lower errors result if both $W_{o}$ and $W_{i}$ have only antistable zeros. Therefore, if $N_{o}$ has no full row rank or has some stable zeros, then an all-pass factor $\widetilde{M}_{o}$ can be determined (see [10] for the continuous-time case and [8] for the discrete-time case) such that

$$
N_{o}=\widetilde{M}_{o}\left[\begin{array}{c}
\widetilde{N}_{o} \\
0
\end{array}\right]
$$

where $\widetilde{N}_{o}$ has full row rank and has only unstable zeros. Similarly, by applying the same procedure to $N_{i}^{T}$ we can determine an all-pass factor $\widetilde{M}_{i}$ such that

$$
N_{i}=\left[\begin{array}{ll}
\widetilde{N}_{i} & 0
\end{array}\right] \widetilde{M}_{i}
$$

where $\widetilde{N}_{i}$ has full column rank and has only unstable zeros. It follows that

$$
\left\|N_{o}\left(G-G_{r}\right) N_{i}\right\|_{\infty}=\left\|\widetilde{N}_{o}\left(G-G_{r}\right) \widetilde{N}_{i}\right\|_{\infty}
$$

which is in the standard form required for the application of the FWHNA Algorithm.

\section{Conclusion}

We derived projection formulas for the two standard formulations of the FWHNA problem and we proposed an implementable algorithm for the solution of the FWHNA problem in the most general setting. The proposed FWHNA algorithm exploits all structural features of the underlying problem and can be employed to solve the frequency-weighted $L_{\infty}$-norm approximation problem without any restrictions on the weights. The proposed FWHNA Algorithm has been implemented in Fortran 77 as a robust numerical software and is freely available in the current release of the systems and control library SLICOT $^{1}$ (see also [3] for a description of SLICOT). The implementation relies on powerful and flexible solvers for various Sylvester equations and Sylvester system which are available in SLICOT and in the linear algebra package LAPACK [1]. User friendly $m$ - and $m e x$-file based interfaces to the new SLICOT software have been also implemented to execute the FWHNA Algorithm under MATLAB.

\section{References}

[1] E. Anderson, Z. Bai, J. Bishop, J. Demmel, J. Du Croz, A. Greenbaum, S. Hammarling, A. McKenney, S. Ostrouchov, and D. Sorensen. LAPACK User's Guide, Third Edition. SIAM, Philadelphia, 1999.

[2] R. H. Bartels and G. W. Stewart. Algorithm 432: Solution of the matrix equation $\mathrm{AX}+\mathrm{XB}=\mathrm{C}$. Comm. ACM, 15:820826, 1972.

[3] P. Benner, V. Mehrmann, V. Sima, S. Van Huffel, and A. Varga. SLICOT - a subroutine library in systems and control theory. In B. N. Datta, editor, Applied and Computational Control, Signals and Circuits, vol. 1, pp. 499539, Birkhäuser, 1999.

[4] K. Glover. All optimal Hankel-norm approximations of linear multivariable systems and their $L^{\infty}$-error bounds. Int. J. Control, 39:1115-1193, 1974.

[5] Y. S. Hung and K. Glover. Optimal Hankel-norm approximation of stable systems with first-order stable weighting functions. Systems \& Control Lett., 7:165-172, 1986.

[6] B. Kågström and L. Westin. Generalized Schur methods with condition estimators for solving the generalized Sylvester equation. IEEE Trans. Autom. Control, 34:745751, 1989.

[7] G. A. Latham and B. D. O. Anderson. Frequencyweighted optimal Hankel norm approximation of stable transfer functions. Systems \& Control Lett., 5:229-236, 1985.

[8] C. Oară and A. Varga. The general inner-outer factorization problem for discrete-time systems. Proc. ECC'99, Karlsruhe, Germany, 1999.

[9] C. Oară and A. Varga. Minimal degree coprime factorization of rational matrices. SIAM J. Matrix Anal. Appl., 21:245-278, 1999.

[10] C. Oară and A. Varga. Computation of general inner-outer and spectral factorizations. IEEE Trans. Autom. Control, 45:2307-2325, 2000.

[11] V. Sima. Comparison of some algorithms for solving Lyapunov type equations. Rev. Roum. Scie. Techn. - Electrotech. et Energ., 25:235-246, 1980.

[12] A. Varga. Explicit formulas for an efficient implementation of the frequency-weighted model reduction approach. Proc. ECC'99, Groningen, NL, pp. 693-696, 1993.

[13] A. Varga. Computation of Kronecker-like forms of a system pencil: Applications, algorithms and software. Proc. CACSD'96 Symposium, Dearborn, MI, pp. 77-82, 1996.

[14] A. Varga. Computation of coprime factorizations of rational matrices. Lin. Alg. \& Appl., 271:83-115, 1998.

[15] A. Varga. Computation of inner-outer factorizations of rational matrices. IEEE Trans. Autom. Control, 43:684688, 1998.

[16] A. Varga and P. Parrilo. Fast algorithms for solving $L_{\infty^{-}}$ norm minimization problems. (submitted to CDC'2001)

[17] K. Zhou. Frequency-weighted $L_{\infty}$ norm and optimal Hankel norm model reduction. IEEE Trans. Autom. Control, 40:1687-1699, 1995.

\footnotetext{
${ }^{1}$ ftp://wgs.esat.kuleuven.ac.be/pub/WGS/SLICOT/libindex.html
} 